DESY-05-005

PC 077.1204

\title{
Exclusive production of pentaquarks in the scaling regime*
}

\author{
M. Diehl ${ }^{\mathrm{a}}$, B. Pire ${ }^{\mathrm{b} \dagger}$ L. Szymanowski ${ }^{\mathrm{c}}$ \\ aDeutsches Elektronen-Synchroton DESY, 22603 Hamburg, Germany \\ ${ }^{\mathrm{b}} \mathrm{CPhT}$, École Polytechnique, 91128 Palaiseau, France \\ 'Soltan Institute for Nuclear Studies, Hoża 69, 00-681 Warsaw, Poland and \\ Université de Liège, 4000 Liège, Belgium
}

We investigate two exclusive reactions with a $\Theta^{+}$pentaquark in the final state: electroproduction of a $K$ meson on the nucleon, and $K^{+}$scattering on a neutron target producing a lepton pair. These reactions offer unique opportunities to investigate the structure of pentaquark baryons at parton level. We discuss the generalized parton distributions for the $N \rightarrow \Theta^{+}$transition and give the leading order amplitude for these processes in the Bjorken regime.

\section{Motivation}

Several experiments have reported evidence for the existence of a narrow baryon resonance $\Theta^{+}$with strangeness $S=+1$, whose minimal quark content is uudd $\bar{s}$ [1]. Triggered by the prediction of its mass and width in 34, the observation of this hadron promises to shed new light on our picture of baryons in QCD. A fundamental question is how the structure of baryons manifests itself in terms of the basic degrees of freedom in QCD, at the level of partons. This structure at short distances can be probed in hard exclusive scattering processes, where it is encoded in generalized parton distributions [56]. In Ref. 7] we introduced the transition GPDs from the nucleon to the $\Theta^{+}$and investigated electroproduction processes where they could be measured, hopefully already in existing experiments at DESY and Jefferson Lab. The crossed channel, where a timelike virtual photon is produced together with the pentaquark, might be studied at intense kaon beam facilities presently under discussion [2].

${ }^{*}$ Talk given at the 10th International Conference on the Structure of Baryons (Baryons 2004), Palaiseau, France, 25-29 October 2004. To appear in the Proceedings.

${ }^{\dagger} \mathrm{UMR} 7644 \mathrm{du}$ CNRS 

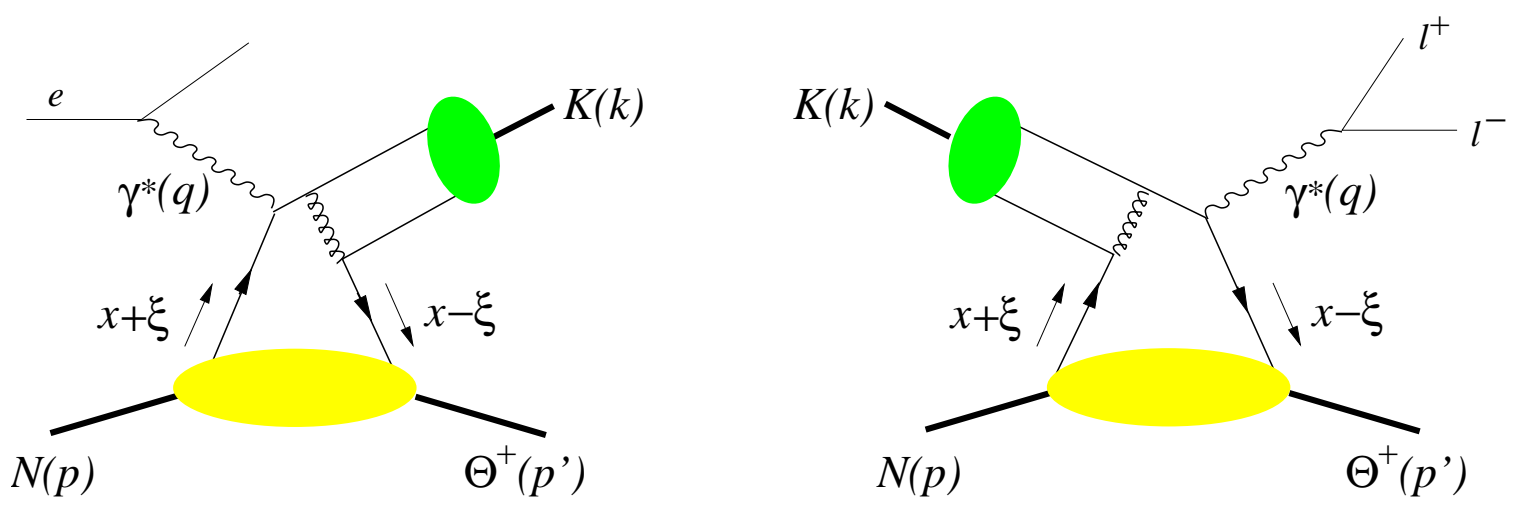

Figure 1. Example diagrams at leading order in $\alpha_{s}$ for pentaquark electroproduction (left) and its timelike counterpart (right) in the scaling limit. In both cases three further diagrams are obtained by attaching the photon to the quark lines in all possible ways. The plus-momentum fractions $x$ and $\xi$ refer to the average momentum $P=\frac{1}{2}\left(p+p^{\prime}\right)$ of nucleon and pentaquark.

\section{Processes}

We consider the processes

$$
\begin{aligned}
e p & \rightarrow e \bar{K}^{0} \Theta^{+}, \quad \text { en } \rightarrow e K^{-} \Theta^{+}, \\
K^{+} n & \rightarrow \ell^{+} \ell^{-} \Theta^{+},
\end{aligned}
$$

where $\ell$ is an electron or muon and where the $\Theta^{+}$subsequently decays into $K^{0} p$ or $K^{+} n$. The case of $K^{*}$ electroproduction was also discussed in Ref. [7. We are interested in the Bjorken limit, which for the electroproduction processes (II) is given by large $Q^{2}=-q^{2}$ at fixed $t=\left(p-p^{\prime}\right)^{2}$ and fixed scaling variable $x_{B}=Q^{2} /(2 p q)$. For the crossed process (2) we take the limit of large $Q^{2}=q^{2}$ at fixed $t=\left(p-p^{\prime}\right)^{2}$ and fixed $\tau=Q^{2} /(2 p k)$. Four-momenta are specified in Fig. 1.

According to the factorization theorem for meson production [8], the Bjorken limit implies factorization of the exclusive amplitudes into a perturbatively calculable subprocess at quark level, the distribution amplitude of the meson, and a generalized parton distribution (GPD) describing the transition from the nucleon to the $\Theta^{+}$(see Fig. 11). The arguments for factorization in [8] do not rely on the photon being spacelike and can be extended to the case $K^{-} n \rightarrow \gamma^{*} \Theta^{+}$with the same nonperturbative input. The dominant polarization of the spacelike or timelike photon is then longitudinal, and the corresponding cross sections have a characteristic scaling behavior as we will see below.

\section{The transition GPDs and their physics}

To define the transition GPDs we introduce light-cone coordinates $v^{ \pm}=\left(v^{0} \pm v^{3}\right) / \sqrt{2}$ and transverse components $v_{T}=\left(v^{1}, v^{2}\right)$ for any four-vector $v$. The skewness variable 
$\xi=\left(p-p^{\prime}\right)^{+} /\left(p+p^{\prime}\right)^{+}$describes the loss of plus-momentum of the incident nucleon and is connected with $x_{B}$ or $\tau$ by $\xi \approx x_{B} /\left(2-x_{B}\right)$ or $\xi \approx \tau /(2-\tau)$ in the Bjorken limit. We assume that the $\Theta^{+}$has spin $J=\frac{1}{2}$, isospin $I=0$ and intrinsic parity either $\eta_{\Theta}=1$ or $\eta_{\Theta}=-1$. The hadronic matrix elements needed at leading-twist accuracy are

$$
\begin{aligned}
& F_{V}=\left.\frac{1}{2} \int \frac{d z^{-}}{2 \pi} e^{i x P^{+} z^{-}}\left\langle\Theta^{+}\left|\bar{d}\left(-\frac{1}{2} z\right) \gamma^{+} s\left(\frac{1}{2} z\right)\right| p\right\rangle\right|_{z^{+}=0, z_{T}=0} \\
& F_{A}=\left.\frac{1}{2} \int \frac{d z^{-}}{2 \pi} e^{i x P^{+} z^{-}}\left\langle\Theta^{+}\left|\bar{d}\left(-\frac{1}{2} z\right) \gamma^{+} \gamma_{5} s\left(\frac{1}{2} z\right)\right| p\right\rangle\right|_{z^{+}=0, z_{T}=0}
\end{aligned}
$$

with $P=\frac{1}{2}\left(p+p^{\prime}\right)$. The corresponding $p \rightarrow \Theta^{+}$transition GPDs are defined by

$$
\begin{aligned}
& F_{V}=\frac{1}{2 P^{+}}\left[H(x, \xi, t) \bar{u}\left(p^{\prime}\right) \gamma^{+} u(p)+E(x, \xi, t) \bar{u}\left(p^{\prime}\right) \frac{i \sigma^{+\alpha}\left(p^{\prime}-p\right)_{\alpha}}{m_{\Theta}+m_{N}} u(p)\right], \\
& F_{A}=\frac{1}{2 P^{+}}\left[\tilde{H}(x, \xi, t) \bar{u}\left(p^{\prime}\right) \gamma^{+} \gamma_{5} u(p)+\tilde{E}(x, \xi, t) \bar{u}\left(p^{\prime}\right) \frac{\gamma_{5}\left(p^{\prime}-p\right)^{+}}{m_{\Theta}+m_{N}} u(p)\right]
\end{aligned}
$$

for $\eta_{\Theta}=1$ and

$$
\begin{aligned}
& F_{V}=\frac{1}{2 P^{+}}\left[\tilde{H}(x, \xi, t) \bar{u}\left(p^{\prime}\right) \gamma^{+} \gamma_{5} u(p)+\tilde{E}(x, \xi, t) \bar{u}\left(p^{\prime}\right) \frac{\gamma_{5}\left(p^{\prime}-p\right)^{+}}{m_{\Theta}+m_{N}} u(p)\right], \\
& F_{A}=\frac{1}{2 P^{+}}\left[H(x, \xi, t) \bar{u}\left(p^{\prime}\right) \gamma^{+} u(p)+E(x, \xi, t) \bar{u}\left(p^{\prime}\right) \frac{i \sigma^{+\alpha}\left(p^{\prime}-p\right)_{\alpha}}{m_{\Theta}+m_{N}} u(p)\right]
\end{aligned}
$$

for $\eta_{\Theta}=-1$, where $m_{N}$ and $m_{\Theta}$ denote the mass of the nucleon and the $\Theta^{+}$, respectively. Due to isospin symmetry the GPDs for the transition $n \rightarrow \Theta^{+}$are equal to those for $p \rightarrow \Theta^{+}$up to a global sign.

As shown in [9], GPDs contain information about the spatial structure of hadrons. A Fourier transform of their dependence on $t$ tells us about the transverse size of the hadrons in question. For instance, for $\xi<|x|<1$ the transverse positions of all partons must match in the proton and the $\Theta^{+}$, including the quark or antiquark taking part in the hard scattering. Thus, the $p \rightarrow \Theta^{+}$transition GPDs probe the partonic structure of the $\Theta^{+}$by requiring its wave function to overlap with the wave function of appropriate configurations in the nucleon.

\section{Scattering amplitude and cross section}

At leading order in $1 / Q$ and in $\alpha_{s}$, the factorization theorem gives scattering amplitudes

$$
\begin{array}{r}
\mathcal{A}\left(\gamma^{*} p \rightarrow \bar{K}^{0} \Theta^{+}\right)=i e \frac{8 \pi \alpha_{s}}{27} \frac{f_{K}}{Q}\left[I_{K} \int_{-1}^{1} \frac{d x}{\xi-x-i \epsilon}\left(F_{A}(x, \xi, t)-F_{A}(-x, \xi, t)\right)\right. \\
\left.+J_{K} \int_{-1}^{1} \frac{d x}{\xi-x-i \epsilon}\left(F_{A}(x, \xi, t)+F_{A}(-x, \xi, t)\right)\right], \\
\mathcal{A}\left(\gamma^{*} n \rightarrow K^{-} \Theta^{+}\right)=-i e \frac{8 \pi \alpha_{s}}{27} \frac{f_{K}}{Q}\left[I_{K} \int_{-1}^{1} \frac{d x}{\xi-x-i \epsilon}\left(F_{A}(x, \xi, t)+2 F_{A}(-x, \xi, t)\right)\right. \\
\left.+J_{K} \int_{-1}^{1} \frac{d x}{\xi-x-i \epsilon}\left(F_{A}(x, \xi, t)-2 F_{A}(-x, \xi, t)\right)\right]
\end{array}
$$


for longitudinal polarization of the photon, independently of the parity of the $\Theta^{+}$. Here $I$ and $J$ are definite integrals over the twist-two distribution amplitude of the kaon, given in [7]. At fixed $\xi$ and $t$ the amplitude scales like $1 / Q$, up to logarithmic corrections from the running of $\alpha_{s}$ and from the scale evolution of the GPDs and distribution amplitudes. The corresponding $e p$ or en cross section is obtained from

$\frac{d \sigma}{d Q^{2} d t d y}=\frac{\alpha_{e m}}{32 \pi^{2}} \frac{1-y}{y} \frac{x_{B}^{2}}{Q^{6}} \sum_{\lambda^{\prime}, \lambda}\left|\mathcal{A}_{\lambda^{\prime}, \lambda}\right|^{2}$,

where $\lambda\left(\lambda^{\prime}\right)$ denotes the helicity of the incoming (outgoing) baryon and where $y$ is the usual inelasticity parameter introduced in deep inelastic scattering. Realistic cross section estimates based on these expressions are unfortunately not possible at present, due to our ignorance of the $p \rightarrow \Theta^{+}$transition GPDs.

The amplitude for the crossed process (2) is obtained in a straightforward way [10]. At equal $Q^{2}, t$ and $\xi$ one has

$\mathcal{A}_{\lambda^{\prime}, \lambda}\left(K^{+} n \rightarrow \gamma^{*} \Theta^{+}\right)=\left[\mathcal{A}_{\lambda^{\prime}, \lambda}\left(\gamma^{*} n \rightarrow K^{-} \Theta^{+}\right)\right]^{*}$

at leading power in $1 / Q$ and in $\alpha_{S}$, up to an irrelevant global phase and provided that the phase convention for helicity spinors gives real valued matrix elements in (44) and (5). We anticipate that the relation (8) no longer holds at the level of corrections in $\alpha_{S}$ or in $1 / Q$. The cross section for the overall process $K^{+} n \rightarrow \ell^{+} \ell^{-} \Theta^{+}$is obtained from

$\frac{d \sigma}{d Q^{2} d t d(\cos \theta)}=\frac{\alpha_{\mathrm{em}}}{128 \pi^{2}} \sin ^{2} \theta \frac{\tau^{2}}{Q^{6}} \sum_{\lambda^{\prime}, \lambda}\left|\mathcal{A}_{\lambda^{\prime}, \lambda}\right|^{2}$,

where $\theta$ is the polar decay angle of the photon in its rest frame (cf. Fig. 5 of [11]). The $\sin ^{2} \theta$ behavior in (91) reflects the purely longitudinal $\gamma^{*}$ polarization. Note that in this process the $\Theta^{+}$is produced in the very clean environment of a final state with only the resonance and a high-mass lepton pair.

\section{Acknowledgments}

This work is supported by the Joint Research Activity "Generalised Parton Distributions" in Integrated Infrastructure Initiative "Hadron Physics" of the European Union, contract No. RII3-CT-2004-506078 and by the Polish Grant 1 P03B 028 28. L.Sz. is a Visiting Fellow of the Fonds National pour la Recherche Scientifique (Belgium). The work of B.P. and L. Sz. is partially supported by the French-Polish scientific agreement Polonium.

\section{REFERENCES}

1. T. Nakano et al. [LEPS Collaboration], Phys. Rev. Lett. 91, 012002 (2003) and contribution to this conference.

2. S. Nagamiya, Nucl. Instrum. Meth. B 214, 216 (2004).

3. D. Diakonov, V. Petrov and M. V. Polyakov, Z. Phys. A 359, 305 (1997).

4. M. Praszalowicz, Phys. Lett. B 575, 234 (2003). 
5. D. Müller et al., Fortschr. Phys. 42, 101 (1994); X. D. Ji, Phys. Rev. Lett. 78, 610 (1997); A. V. Radyushkin, Phys. Rev. D56, 5524 (1997).

6. K. Goeke, M. V. Polyakov and M. Vanderhaeghen, Prog. Part. Nucl. Phys. 47, 401 (2001); M. Diehl, Phys. Rept. 388, 41 (2003).

7. M. Diehl, B. Pire and L. Szymanowski, Phys. Lett. B 584, 58 (2004).

8. J. C. Collins, L. Frankfurt and M. Strikman, Phys. Rev. D 56, 2982 (1997).

9. M. Burkardt, Phys. Rev. D 62, 071503 (2000), Erratum-ibid. D 66, 119903 (2002);

J. P. Ralston and B. Pire, Phys. Rev. D 66, 111501 (2002); M. Diehl, Eur. Phys. J. C 25, 223 (2002), Erratum-ibid. C 31, 277 (2003).

10. E. R. Berger, M. Diehl and B. Pire, Phys. Lett. B 523, 265 (2001).

11. E. R. Berger, M. Diehl and B. Pire, Eur. Phys. J. C 23, 675 (2002). 